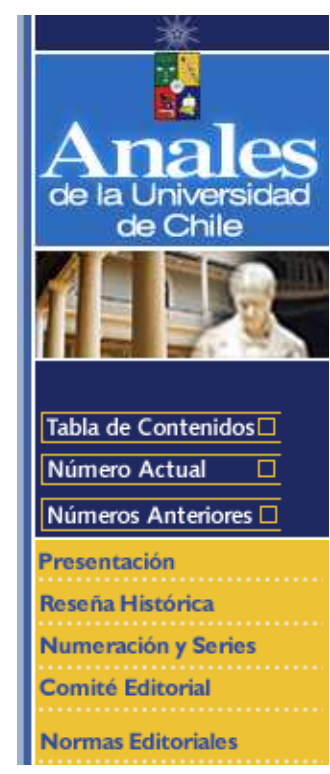

Anales de la Universidad de Chile Sexta Serie, No11, agosto 2000

Tabla de Contenidos $\square$

Número Actual $\square$

Numeración y Series

Comité Editorial

Normas Editoriales

Antes dela Universidadechie Sexta Serie, No11, agosto 2000 


\title{
- Microbiología
}

\section{[La microbiología y los alimentos]}

\author{
López V. , Luis, Prof. y Avendaño V. Sonia
}

Facultad de Ciencias Químicas y Farmacéuticas, Universidad de Chile

\Cita / Referencia

López V.,Luis y Avendaño V.,Sonia. La microbiología y los alimentos. Anales de la Universidad de

Chile. VI serie: No11, agosto 2000

D http://www2.anales.uchile.cl/CDA/an_completa

/0,1281,SCID\%253D3500\%2526ISID\%253D7\%2526ACT\%253D0\%2526PRT\%253D3499,00.html

\section{- Texto}

En la actualidad, el comercio internacional de alimentos representa una actividad cada vez más importante en la provisión de regímenes alimentarios inocuos y nutritivos para las poblaciones del mundo. Este comercio internacional genera además un beneficio doble, no solamente introduce una mayor variedad de productos en la oferta alimentaria aumentando las posibilidades de elección de los consumidores, sino que también proporciona divisas a los países exportadores.

Sin embargo, esta situación ha aumentado las posibilidades de transmisión de agentes infecciosos y pone de relieve la necesidad de adoptar un criterio internacional, para estimar el riesgo potencial de estos patógenos microbianos y establecer las medidas apropiadas para su reducción y/o eliminación. Más de tres millones de personas mueren anualmente de enfermedades diarreicas, mientras que cientos de millones padecen episodios frecuentes de diarrea y sus consecuencias debilitantes. Son motivo de preocupación especial, los alcances y la naturaleza potencialmente mortal de tales enfermedades en los grupos sensibles de la población (infantes, ancianos, embarazadas e inmunodeprimidos) principalmente de los países en desarrollo. El mundo ha experimentado un aumento continuo de la incidencia de las enfermedades transmitidas por los alimentos (ETA) y es probable que la carga mayor de ellas recaiga sobre los países en desarrollo.

Junto a esto es necesario considerar grandes pérdidas económicas, por alteración de alimentos causada por el desarrollo de microorganismos de carácter alterador. Esto afecta no sólo a la exportación de productos alimenticios, sino que también a nivel regional, donde además del costo monetario que esto implica, se disminuye considerablemente la oferta de alimentos, como por ejemplo en regiones tropicales donde las condiciones ambientales favorecen el desarrollo de hongos, los que además de causar alteraciones pueden elaborar metabolitos tóxicos (micotoxinas) en productos como cereales y granos en general.

Es en este contexto, que la Microbiología de los Alimentos se ha transformado en una herramienta de gran utilidad y se perfila como una ciencia en evolución permanente destinada a solucionar los problemas anteriormente mencionados, a través de metodologías cada día más avanzadas y eficaces.

Se podría considerar que esta disciplina comenzó como una rama de la Microbiología General, alrededor del año 1800 al demostrarse la relación entre los alimentos y los microorganismos. En 1837, Schwann propuso que las levaduras que aparecian durante la fermentación alcohólica eran plantas microscópicas; entre 1857 y 1876 , Pasteur demostró que los microorganismos eran los responsables de los cambios químicos que ocurrían en los alimentos y algunas bebidas. Sus observaciones establecieron las bases para el desarrollo de la actual Microbiología de Alimentos.

La evolución de esta disciplina a nivel mundial ha sido constante. En la actualidad existen en Chile un gran número de organismos e instituciones estatales y privadas que se dedican a esta rama de la Microbiología, entre las cuales se encuentra la Facultad de Ciencias Químicas y Farmacéuticas de la Universidad de Chile. En sus comienzos, el estudio de la contaminación de los productos alimenticios formaba parte de la Asignatura de Microbiología General impartida a los alumnos de las carreras de Química y Farmacia y Bioquímica. Posteriormente, durante la década del 70, al crear la Carrera de Ingeniería en Alimentos, esta disciplina se transformó en una asignatura obligatoria para sus alumnos, siendo su primer Profesor la Dra. Silvia Mendoza G., con la colaboración de la Prof. Sonia Avendaño V. y posteriormente del Prof. Luis López V. En dicha ocasión se elaboró un completo programa teórico-práctico, considerando las necesidades más inmediatas de la Industria de Alimentos nacional, proyectado ya en esos momentos, a lo que sería el comercio internacional de alimentos.

A nivel latinoamericano, en la década del 60 se formó en Perú el Centro Latinoamericano de Enseñanza e Investigación en Bacteriología Alimentaria (CLEIBA), organismo de reconocido prestigio como núcleo formador de profesionales de la región centro y sudamericana relacionados con el área de la Microbiología e Higiene de los Alimentos. Sus aportes fueron de gran ayuda, para los profesionales chilenos que comenzaban su labor en esta área, no sólo aportando valiosa información en su formación teórica, sino que también a nivel de laboratorios de control e investigación.

A nivel mundial, en la misma época se creaba la International Commission on Microbiological Specifications for Foods (ICMSF), la cual hasta el momento es el organismo de excelencia en el tema. Su aporte, distribuyendo información a través de la publicación de textos de consulta y apoyo tanto para la docencia e investigación a nivel académico, como también contribuyendo con especificaciones microbiológicas, medidas de prevención y de control de gran utilidad para la industria de alimentos, es de reconocido prestigio. Este organismo se compone de diferentes comisiones 
representando diversas regiones del globo terráqueo. Chile forma parte del Latin-American Subcommission (LAS) y está representado por la Dra. Eliana Marambio L. (Instituto de Salud Pública de Chile).

En Chile, la difusión e intercambio de experiencias en esta área, tanto de tipo teórico como práctico, se ha realizado desde los inicios de esta disciplina a través de cursos, seminarios, talleres, mesas redondas y congresos.

El Dr. Hermann Schmidt-Hebbel fue quizás el pionero y mayor impulsor en la organización y coordinación de cursos, incorporando al tema de la Tecnología de Alimentos aspectos microbiológicos de utilidad para la industria de alimentos, como por ejemplo tópicos relacionados con las enfermedades transmitidas por alimentos, características de patógenos bacterianos, significado de los hongos y sus metabolitos tóxicos presentes en productos alimenticios, alteradores de origen microbiano, desarrollo de resistencia debido a la incorporación de antibióticos en alimentos y especificaciones microbiológicas a nivel nacional e internacional, entre otros.

Los Congresos nacionales también han permitido el avance de esta disciplina, ya que siempre ha formado parte de la temática de eventos tales como Congresos Chilenos de Nutrición, Bromatología y Toxicología, de Microbiología y de Tecnología de Alimentos. A nivel internacional es reconocida la activa participación de los microbiólogos de alimentos en eventos regionales, latinoamericanos y mundiales. En 1987 se realizó en Buenos Aires, Argentina con el auspicio de la ICMSF, el 1er Congreso Latinoamericano de Microbiología e Higiene de Alimentos, el que ha continuado desarrollándose cada 2 años en países tales como Venezuela, Uruguay, Perú y Brasil. Para el año 2002 Chile ha comprometido, a través del LAS, su participación como país organizador de este prestigioso evento.

A pesar de todos los esfuerzos realizados, destinados a disminuir el riesgo potencial que implica la presencia de microorganismos en productos alimenticios, no se ha logrado detener o disminuir en forma adecuada la incidencia de las ETA. Más aún, factores tales como el aumento explosivo de la población y sus comportamientos o hábitos de la población, los cambios tecnológicos a nivel de industrias de alimentos, el aumento considerable en el transporte de la población y de los alimentos, la adaptación de algunos microorganismos al ambiente y el desarrollo de resistencia a antimicrobianos han contribuido a producir cambios significativos en la epidemiología de las ETA, como por ejemplo la aparición de microorganismos denominados patógenos emergentes. No se trata de agentes microbianos nuevos o recientemente descubiertos, sino que no se había demostrado su participación como patógenos que pudieran ser transmitidos por los alimentos. Entre ellos se pueden mencionar a Escherichia coli 0157:H7, Salmonella enreritidis, Campylobacter jejuni, Virus norwalk, Listeria monocyrogenes, Vibrio vulnificus, Clostridium butyricum y otros. Su participación en el desarrollo de brotes a nivel mundial está claramente demostrada, en Chile ya se ha detectado su presencia en una gran variedad de alimentos, L. monocytogenes en productos lácteos, E. coli 0157: H7 en carne molida, C. jejuni en aves y $S$. enteritidis en huevos.

Considerando lo mencionado anteriormente, no sólo sería necesario establecer medidas preventivas y de control como posible solución al problema, sino que también es imprescindible efectuar acciones de inspección y vigilancia para que estas medidas se lleven a cabo en forma efectiva. A nivel nacional, el Ministerio de Salud mantiene un programa de vigilancia de la situación en la Región Metropolitana y en las diferentes regiones del país. Inicialmente contó con la participación del Instituto de Salud Pública, donde se realizaban diariamente los análisis microbiológicos y químicos de una determinada cantidad de muestras obtenidas por inspectores en diferentes locales de elaboración, expendio o consumo de alimentos de la Región Metropolitana. Posteriormente, esta labor de control fue asumida por el Laboratorio Central del Servicio de Salud Metropolitano del Ambiente (SESMA), organismo que tiene en estos momentos la responsabilidad de planificar, inspeccionar, controlar y sancionar las posibles no conformidades detectadas en los muestreos.

Un gran avance en la solución de la problemática planteada, ha sido la revisión y actualización del antiguo Reglamento Sanitario de Alimentos. Con respecto a las especificaciones microbiológicas que deben cumplir los alimentos, el nuevo documento presenta requisitos acordes a lo que se exige a nivel internacional, pero planteados de acuerdo a la realidad nacional. Para ello se contó durante su estudio, con la participación de representantes de industrias de alimentos de todos los rubros, quienes a través de datos experimentales debidamente documentados, demostraron el nivel de calidad que podian alcanzar sus productos, lo cual fue fundamental para establecer los límites exigidos en el actual documento. Entre las ventajas que presenta este reglamento se pueden citar la incorporación de un gran número de grupos de alimentos, subdivididos en subgrupos de acuerdo al proceso tecnológico aplicado durante su elaboración; se presentan además las categorías en la que cada subgrupo de alimento puede ser clasificado; se aumenta el número de muestras a analizar de 1 a 5 , permitiendo de esta forma hacer más representativo el control; se modifica y disminuye el tipo de parámetros microbiológicos a determinar de acuerdo al tipo de alimento y finalmente se permite la presencia en determinados niveles de microorganismos tales como Escherichia coli y Staphylococcus aureus, modificando la especificación de ausencia establecida en el antiguo documento.

Debido a la gran diversidad de metodologías utilizadas en la ejecución de los análisis microbiológicos y como complemento técnico del nuevo Reglamento Sanitario de los Alimentos, el Ministerio de Salud patrocinó la confección de un Manual de Técnicas Microbiológicas para Alimentos y Aguas, en la cual participaron representantes de instituciones estatales y privadas. El documento elaborado fue publicado en el año 1998 y ha sido de gran utilidad para los laboratorios estatales y privados dedicados al control microbiológico de alimentos, permitiéndoles estandarizar la elección de medios de cultivo, preparación de las muestras para el análisis, procedimientos de ensayo e interpretación de los resultados obtenidos.

Con el incremento del comercio internacional, sobre todo de productos marinos, los países importadores han establecido ciertas especificaciones que deben cumplir las industrias participantes. Es así como la Comunidad Económica Europea, establece que las plantas elaboradoras que exportan productos marinos deben tener implementado un Sistema de Aseguramiento de Calidad, el cual es supervisado en Chile por el Servicio Nacional de Pesca (SERNAPESCA). Existe al respecto una partición activa de Laboratorios de Control Microbiológico privados o estatales del país, que verifican la calidad de los productos exportados. Estos Laboratorios deben ser acreditados a través de la División de Acreditación del Instituto Nacional de Normalización (INN). El proceso de acreditación propiamente tal, es efectuado por un equipo de especialistas en base a la revisión de los documentos que componen el sistema de calidad del laboratorio en cuestión, junto con las respectivas auditorías en terreno, todo esto de acuerdo a normativas internacionales tales como la Guía ISO/IEC 25.

Son varios los avances que en este último tiempo se han desarrollado, tendientes a facilitar la labor de control y 
prevención de los contaminantes de origen microbiológico:

* Se han propuesto sistemas tales como el Longitudinal Integrated Safety Assurance (LISA), Just In Time (JIT), Sanitary Assessment of Food Environment (SAFE) y Self Care Action Program (SCAP), pero quizás el más conocido y de resultados documentadamente comprobados es el Hazard Analysis Critical Control Points (HACCP). Este sistema comenzó en 1959 cuando a la firma Pillsbury Company de EEUU se le solicitó proveer de alimentos al programa espacial, que presentaran «cero defectos o peligros». Sin embargo, éste no es un sistema que implique riesgo cero, pero ha sido diseñado para minimizar el riesgo de introducir peligros al alimento. Su uso a nivel industrial se presenta como una eficaz herramienta, dado que se trata de un concepto científico, racional y sistemático para la identificación y evaluación de peligros potenciales y riesgos asociados con la manufactura, distribución y uso de un producto alimenticio, así como la definición de las medidas preventivas para su control.

* El uso de la llamada Tecnología de Obstáculos (Hurdle Effect), basada en establecer obstáculos o vallas para detener el desarrollo de los microorganismos presentes o que potencialmente vayan a entrar en contacto con un alimento. El manejo o modificación de factores de crecimiento microbiano (obstáculos) tales como pH, actividad de agua, temperatura, concentración de sal, oxígeno y preservantes, entre otros permite dificultar el desarrollo de los microorganismos, a través de una combinación de tal forma que se establezca una suerte de efecto sinérgico entre todos ellos, lo cual es de mayor efectividad que la modificación o ajuste de cada uno de ellos por separado.

* Los programas computacionales que permiten predecir el destino de un determinado microorganismo o grupo de ellos, a través de simulaciones (respaldadas con experiencias de laboratorio) en las cuales se modifican determinados parámetros que afectan el crecimiento microbiano, ha servido de base para la llamada Microbiología Predictiva. Se establecen de esta forma modelos matemáticos, que son de gran utilidad sobre todo al predecir el comportamiento de un determinado microrganismo o grupo de ellos al desarrollar nuevos productos.

* El constante desarrollo de metodologías analíticas para la determinación de agentes microbianos o de sus metabolitos, a través de métodos rápidos que facilitan las determinaciones a nivel de planta elaboradora o de laboratorio de control. Es amplia la oferta de kits o dispositivos para análisis basados en métodos que utilizan sustancias cromógenas (MUG), inmunoensayo (ELISA), bioluminiscencia. (ATP+luciferasa), amplificación de trozos de DNA (PCR), inmunoensayo + cromatografía (REVEALR), inmunocaptura (métodos inmunomagnéticos), epifluorescencia, medición de conductividad o de impedancia, fagodetección, etc. La mayoría de ellos han sido validados y recomendados para ser usados en reemplazo de los métodos tradicionales, otros aunque no validados prestan gran ayuda en la industria, sobre todo en circunstancias en que es necesario contar con información rápida y global de la situación de alguna materia prima, superficies de trabajo o higiene de equipos, por ejemplo.

En los últimos años, tanto los gobiernos como los organismos internacionales han recurrido cada vez más a una nueva herramienta, la evaluación de riesgos, con el propósito de establecer un sistema que les permita examinar la información científica y los temas relacionados con la inocuidad de los alimentos.

Esta evaluación de riesgos proporciona un método estructurado y transparente para organizar y evaluar los datos y la información, así como un medio para medir la incertidumbre inherente a los resultados. Anteriormente la evaluación de los riesgos en alimentos se limitaba principalmente a la determinación de los niveles de ciertos productos químicos que podían estar presentes en los alimentos, sin tener una repercusión adversa sobre la salud pública. En el pasado, se consideraba que los temas relacionados con la inocuidad microbiológica de los alimentos eran demasiado complejos para que se pudiera establecer límites de inocuidad cuantitativos basados en una evaluación de riesgos. Sin embargo, los adelantos recientes en la microbiología y los modelos predictivos han conducido a métodos que facilitan la evaluación microbiológica cuantitativa.

Existe una variedad de métodos disponibles para llevar a cabo evaluaciones de riesgos microbiológicos, pero en general ellos pueden ser clasificados dentro de dos categorías amplias: cualitativos o cuantitativos. Como ejemplo se pueden citar el estudio de la presencia de Salmonella enteritidis en huevos y en productos derivados de los mismos en EEUU y de Campylobacter jejuni en pollos en el Canadá. Estos documentos han puesto de relieve la utilidad de la evaluación de riesgos en cuanto proporciona un fundamento científico para la formulación y evaluación de opciones de gestión de ellos a fin de disminuir el riesgo de los agentes patógenos transmitidos por los alimentos.

Atendiendo a esto, la Organización de las Naciones Unidas para la Agricultura y la Alimentación (FAO) y la Organización Mundial de la Salud (OMS) celebraron una Consulta Mixta FAO/OMS de Expertos en la Evaluación de los Riesgos Asociados a los Peligros Microbiológicos en los Alimentos, en Ginebra (Suiza) entre el 15 y 19 de marzo de 1999, donde se plantearon los siguientes objetivos:

* examinar el conocimiento científico actual sobre la evaluación de los riesgos microbiológicos en los alimentos

* recomendar a la FAO, a la OMS y a sus países miembros una estrategia y un enfoque generales para evaluar los riesgos asociados a los peligros microbiológicos

* recomendar metodologías de evaluación de riesgos apropiadas para ser usadas en el nivel internacional a fin de calcular los peligros microbiológicos que entrañan para la salud de los seres humanos

* recomendar la manera en que la FAO, la OMS y sus países miembros pueden desarrollar y utilizar las opciones de gestión de riesgos y

* proponer temas que tengan prioridad en la evaluación de riesgos.

Entre las actividades recomendadas por esta Consulta, se menciona la transferencia a los países en desarrollo de la tecnología necesaria para la evaluación de riesgos microbiológicos. Se establece, además, que «la FAO, la OMS y los países miembros con la participación bilateral de personal especializado, deberían ayudar a los países en desarrollo proporcionándoles la asistencia, la capacitación y el asesoramiento técnico apropiado». Junto a esto, se acota que «los países en vías de desarrollo y los países desarrollados deberían realizar estudios de casos en colaboración, a fin de intercambiar sus conocimientos y experiencia en relación a la evaluación de riesgos microbiológicos».

Como conclusión se puede señalar, que la Microbiología de Alimentos constituye una especialidad de amplia proyección y de gran necesidad tanto para la salud pública, como para la economía de un país.

Es necesario por lo tanto, establecer una estrecha colaboración entre Autoridades Sanitarias, Universidades e 
Industrias de Alimentos, para enfrentar eficazmente el desafío que significa la producción de alimentos seguros.

\section{: Bibliografía}

1. GARBUTT, J. Essentials of Food Microbiology.. Arnold Publisher, London, 1997.

2. INTERNATIONAL COMMISSSION ON MICROBIOLOGICAL SPECIFICATIONS FOR FOODS (ICMSF). Microorganisms in Foods. 6. Microbial Ecology of Food Commodities, Blackie Academic \& Professional, London, 1998.

3. FAO/OMS. Informe de la Consulta Mlixta de Expertos «Evaluación de los Riesgos Microbiológicos presentes en los Alimentos». FAO/OMS, 1999.

4. LAMMERDING, A.M. Y PAOLI, G.M. Quantitative Risk Assessment: An Emerging Tool for Emerging Foodborne Parhogens. Emerging Infectious Diseases 3(4):483-487, 1997.

5. FINAL REPORT «Salmonella enteritidis Risk Assessment: Shell Eggs and Egg Products. The Food Safety and Inspection Service, 1998.

Texto | Bibliografía | Versión Completa (Imprimir) 